\title{
Análisis de la Vegetación en Cronosecuencias Dunares Campo de Dunas Santo Domingo - El Yali
}

\author{
Raúl Caldichoury Rodríguez \\ Departamento de Geografía \\ Universidad de Chile \\ Santiago, Chile \\ e-mail: raul.caldichoury@geotecnica.cl
}

\begin{abstract}
RESUMEN
En este trabajo se analizó el grado de similitud en la estructura de las comunidades de plantas entre dunas de distintos tipos y generaciones en el campo de dunas Santo Domingo-El Yali en la costa de Chile central. Las formas analizadas son correspondientes con distintas fases de una cronosecuencia dunar. El patrón general para los valores de riqueza y cobertura de especies aumenta conforme se avanza en la cronosecuencia. El análisis de agrupamiento, utilizando el coeficiente de similitud de la estructura comunitaria, separa las comunidades de plantas del sistema dunario en dos grandes grupos. El primer grupo reúne las microdunas y cordón paralelo actual con un $50 \%$ de similitud y los cordones paralelos modernos y medios con un $90 \%$ de similitud. El segundo grupo reúne comunidades con coeficientes de similitud inferiores a un $40 \%$, definiéndose tres subgrupos correspondientes a dunas parabólicas medias y antiguas, dunas upsiloidales y cordón de gravas de generación antigua. En general, los valores de similitud son decrecientes en el sentido de la cronosecuencia. Considerando estos resultados, es posible concluir que existen comunidades particulares a los diferentes estadios de la cronosecuencia, particularmente en las fases de evolución de secundarias, es decir, de dunas parabólicas y upsiloidales. Sucesiones del tipo primarias ocurren en dunas primarias, con especies pioneras como Ambrossia chamissonis y Carpobrotus aequilaterus que facilitan el establecimiento de especies más tardías. Las sucesiones secundarias en dunas de tipo secundarias evidencian la influencia de la morfogénesis en el curso de la sucesión.
\end{abstract}

Palabras clave: continuum, trasmudación, cronosecuencia dunar, similitud comunitaria, sucesión.

\section{Vegetation Analysis in Dune Chronosecuences Santo Domingo-EI Yali Dune Field}

\begin{abstract}
This work analyse the similarity of plant community structure between dunes of different kinds and ages in the dune field of Santo Domingo-El Yali, in coastal central Chile, all of which belong to different phases of a dune chronosequence. The general pattern for species richness and cover values increase with the chronosequence. The cluster analysis based on the community structure coefficient of similarity results in two main groups of plant communities. The first group lumps together the microdunes and the present foredune with a $50 \%$ of similarity, and the modern and middle parallel dune ridges with a $90 \%$ of similarity. The second group lumps communities with similarity coefficients lower than $40 \%$. This group has three subgroups including medium and ancient parabolic dunes, upsiloidal dunes, and ancient gravel ridge. In general, values of similarity decrease with the chronosequence. According to the results, it is possible to conclude that there are specific plant communities to each phase of the chronosequence, especially to the parabolic an upsiloidal dune phases. Primary successions occurrs in primary dunes with pioneer species like Ambrossia chamissonis and Carpobrotus aequilaterus, which would allow the establishment of later species. Secondary succession on secondary dunes reflects the influence of morphogenesis on the course of the succession.
\end{abstract}

Key Words: Continuum, transmudación, dune chronosequence, community similarity, succession. 


\section{INTRODUCCIÓN}

Los procesos asociados al cambio de forma de las dunas han sido tratados sistemáticamente a través del concepto de trasmudación (ARAYAVERGARA 1986). Este concepto indica que los cambios de forma de las dunas, se presentan como una sucesión de estadios dunares a la manera de un continuum de cambios morfológicos. En este sentido, los campos de dunas constituyen una importante herramienta para el estudio de la dinámica de la vegetación, ya que en ellos no sólo se disponen de ejemplares diferenciados en su forma, sino también en lo referente a su edad, condición que permite definir cronosecuencias (sustitución del tiempo por el espacio, PICKETT 1989).

En el estudio de la dinámica de la vegetación en dunas, la cronosecuencia se ha aplicado principalmente a sucesiones primarias, es decir, a la vegetación desarrollada sobre nuevos sustratos. Por otro lado, las sucesiones secundarias, es decir, los reemplazos de vegetación preexistente debido a perturbaciones que la destruyen, han sido mayoritariamente estudiadas en campos agrícolas abandonados (ARMESTO y PICKETT 1986); ambos tipos de sucesiones probablemente forman parte de un continuum, cuya manifestación depende fundamentalmente, de las características y duración de las perturbaciones que causan el inicio de la sucesión (WALKER y CHAPIN 1987).

Las causas del cambio de la vegetación han sido un tema central en ecología vegetal. PICKETT et al. (1987) proponen una jerarquía de 3 niveles de causas para explicar la sucesión: a) el primer nivel reúne los más amplios y menos definidos fenómenos (por ejemplo, la disponibilidad de sitio para la sucesión), b) el segundo nivel agrupa los mecanismos de cambio o causas del primer nivel (por ejemplo, escala de la perturbación), c) el tercer nivel corresponde a los factores que determinan el resultado de los procesos del segundo nivel o intermedio (por ejemplo, extensión, tiempo, magnitud). Estos tres niveles jerárquicos han permitido seleccionar las causas apropiadas para explicar la sucesión en relación al nivel de organización analizado.

Los estudios centrados en la caracterización de sucesiones primarias en dunas (OLSON 1958; VAN DER MAAREL et al.1985; LICHTER 1998), no han tratado en toda su dimensión el estudio de la relación entre los cambios estructurales de las comunidades de plantas y la dinámica particular de perturbaciones de los campos dunares como causas generales de sucesiones, y de esta manera no han considerado en forma sistemática la influencia de los mecanísmos geomorfológicos como procesos generadores de cambios ecológicos.

No obstante su utilidad, la cronosecuencia opera con el concepto de condiciones ambientales similares (por ejemplo, clima y disponibilidad de propágulos), para cada estadio, hecho que en particular en ambientes dunares, restringe su utilidad sólo para propósitos de definiciones cualitativas, y para la generación de hipótesis explicativas (PICKETT 1989).

De acuerdo con ARAYA-VERGARA (1989), en el sistema dunar Santo Domingo-El Yali (Fig. 1), es posible reconocer al menos tres generaciones de dunas y cordones de gravas: actual, media y antigua. Las dunas de generación actual, presentan arenas de características similares a las de generación media pero con menores cantidades de limoarcilla $(0,5-0,8 \%)$. Las dunas de generación media, no presentan suelos, tienen contenidos de limo-arcillas entre 0,5-2,0\% y sus arenas son menos oxidadas que las de generación antigua. Las dunas antiguas se caracterizan por presentar suelos con 2 a 3 horizontes y significativas cantidades de limo-arcilla (1-9 $\%$ ), sus arenas tienen los mayores grados de oxidación.

Con respecto a los tipos de dunas, el sistema de Santo Domingo (CALDICHOURY 1990) está constituido por un sistema de cinco cordones paralelos de generaciones, actual y media, a la que se suma una generación moderna, cronológicamente intermedia entre la actual y media. Los cordones están transgredidos por blow-outs, dunas parabólicas y dunas transgresivas. Entre los cordones se han desarrollado depresiones interdunares, en las que es posible observar cordones de gravas, que constituyen depósitos característicos tanto de este sistema como de El Yali.

El sistema El Yali, por su parte, está constituído por un mayor número de formas dunares que 
el sistema de Santo Domingo las que pertenecen a las generaciones actual, media y antigua (CALDICHOURY 1990). Estas mismas generaciones se aplican a los cordones de gravas existentes en este sistema. Los tipos de dunas reconocidos son los siguientes: duna anterior (foredune), blow-outs, dunas paralelas, upsiloidales y longitudinales, barjanes, barjanoides, barjanes coalescentes, dunas transversales y dunas transgresivas.

Observaciones en la costa de Chile central y norte (ARAYA-VERGARA 1986), permiten establecer el siguiente sistema de categorias de dunas para el campo dunar de Santo Domingo-El Yali: a. Dunas primarias: duna anterior (foredune) y dunas paralelas. b. Dunas secundarias: deflación de duna anterior o dunas paralelas y desarrollo de blow-outs; coalescencia de barjanes generando dunas transversales. c. Evolución de secundarias: blow-outs cambiando a dunas parabólicas, elongación de parabólicas generando dunas upsiloidales y longitudinales; coalescencia de

FIgURA 1

a

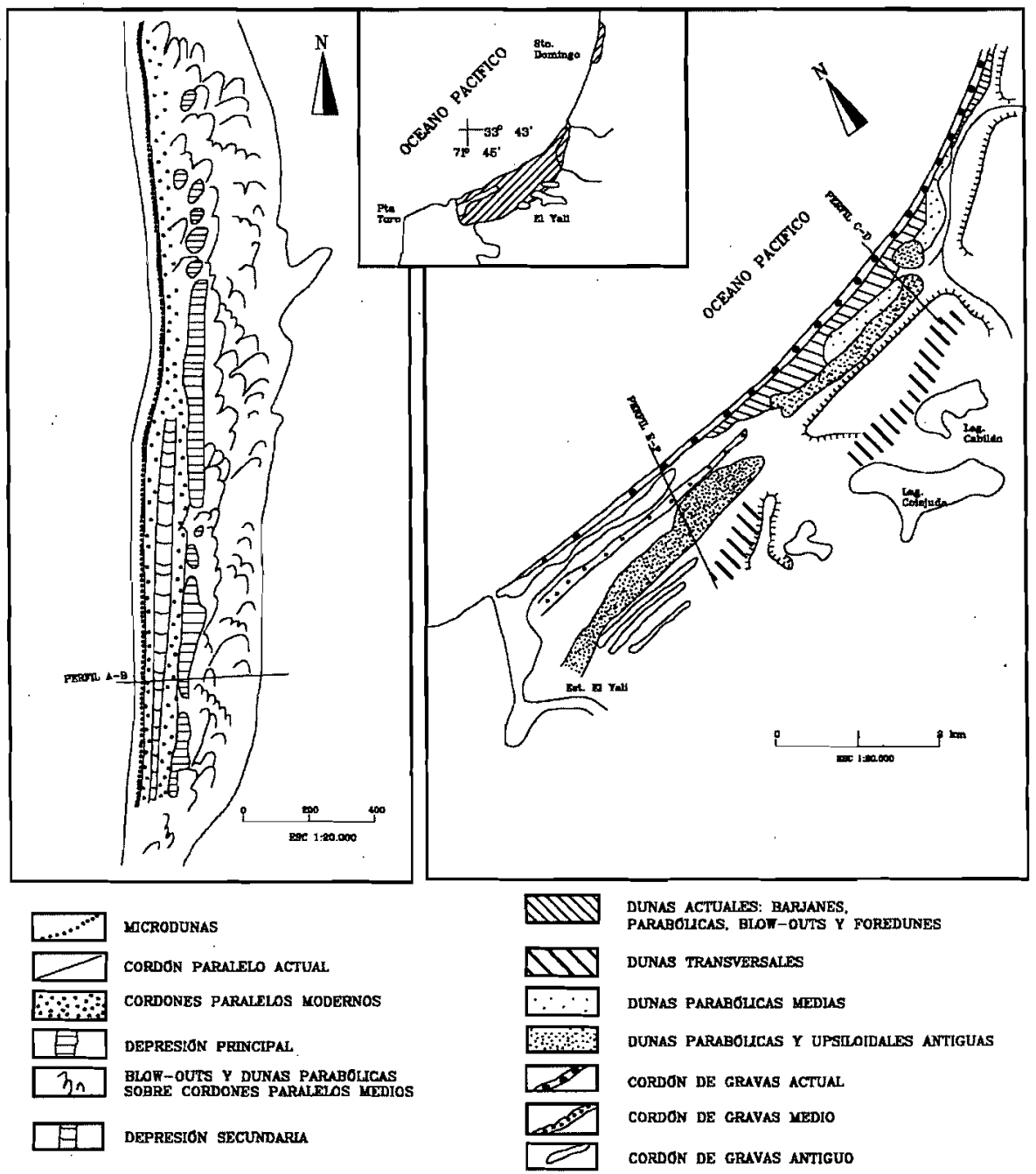

Fig. 1. Campo de dunas Santo Domingo-El Yali, Chile Central. a) Sistema Santo Domingo, b) Sistema EI Yali.

Fig. 1. Santo Domingo-El Yali dune field. a) Santo Domingo system, b) El Yali system. 
parabólicas cambiando a dunas transgresivas

De acuerdo con lo anterior, el propósito de este estudio es establecer: a) el grado de similitud en la estructura de comunidades de plantas de dunas correspondientes a distintas fases del continuum de evolución geomorfologica (cronosecuencia) y b) proponer una interpretación ecológica y geomorfológica de estos cambios.

\section{MATERIALES Y METODOS}

El estudio fue realizado en el campo de dunas de Santo Domingo-El Yali en el litoral de Chile. El sistema de Santo Domingo se encuentra situado, aproximadamente cinco kilómetros al norte del sistema mayor El Yali (Fig. 1). Desde el punto de vista geomofológico y topográfico se reconocen dos unidades mayores sobre la que se han desarrollado ambos sistemas dunares: la primera corresponde a un nivel con alturas entre 2 y $3 \mathrm{~m}$ donde se localizan mayoritariamente las dunas analizadas; la segunda a una terraza costera compuesta de areniscas marinas, con alturas entre 50 y 60 m.

La cronosecuencia dunar en la que se estudiará la similitud en la estructura comunitaria corresponde a: microdunas, cordón paralelo actual, cordón paralelo moderno a medio, dunas parabólicas medias, dunas parabólicas antiguas y dunas upsiloidales antiguas. Los primeros cuatro estadios están representados en el sistema Santo Domingo y los restantes en el sistema El Yali. Sumado a estos tipos y generaciones de dunas se incluye la vegetación del cordón de gravas de generación antigua en el sistema El Yali. Aunque este cordón de gravas no forma parte de la cronosecuencia analizada, el análisis de las comunidades vegetales asociadas a este, permitirá profundizar en mejor medida la relación entre los tipos y características de las formas respecto a la vegetación que en ellos se desarrolla.

Se realizaron dos campañas de terreno durante el período de invierno- primavera de 1989. Durante la primera se hizo un reconocimiento general del área, identificando los tipos y generaciones de dunas de acuerdo a fotografías aéreas y cartografía. De este primer reconocimiento se constató que dunas secundarias actuales no presentan vegetación por lo que no fueron muestreadas. En la segunda campaña, por lo tanto, se muestrearon dunas con vegetación, seleccionando al azar dos ejemplares de duna por cada tipo y generación. El muestreo se realizó utilizando cuadrángulos de $1 \mathrm{~m} 2$, estimando el porcentaje de superficie cubierta por cada especie con respecto al total de la superficie. Los muestreos florísticos se realizaron en los siguientes tipos y generaciones de dunas y cordón de gravas:

- Duna anterior (foredune) y cordones paralelos $(\mathrm{MIC}=$ microdunas. $\mathrm{CPA}=\mathrm{Cordón}$ paralelo actual. $\mathrm{CPMO}=$ cordón paralelo moderno. CPME = cordón paralelo medio).

- Dunas parabólicas ( $\mathrm{PA}=$ parabólicas medias. $\mathrm{PA}=$ parabólicas antiguas).

- Dunas upsiloidales (ULA = upsiloidales antiguas).

- Cordón de gravas ( $\mathrm{CG}=$ cordón de gravas antiguo).

El porcentaje de similitud entre la estructura de las comunidades de cada duna, se estableció mediante el Coeficiente de Similitud de Horn (HORN 1966), definido como:

$$
R o=\frac{S[(X i j+X i k) \log (X i k)]-S(X i j \log X i j)-S(X i k \log X i k)}{[(N J+N K) \log (N J+N K)]-(N J \log N J)-(N K \log N K)}
$$

donde Ro es el índice de similitud para las muestras j y k; X ij y X ik corresponden a la cobertura de la especie $i$ en la muestra $j \mathrm{y} \mathrm{k}$; $\mathrm{Nj}$ corresponde a la proporción total de individuos en la muestra $\mathrm{j}$ y $\mathrm{Nk}$ corresponde a la proporción total de individuos en la muestra k.

Para el agrupamiento de las muestras se utilizó la técnica de Average Linkage Clustering, 
Perfil Fitogeográfico Santo Domingo

$$
\text { (A-B) }
$$
Escala Vertical $1: 1.000$
Escala Hortzental $1: 0.000$

Perfil Fitogeográfico El Yali Centro Norto (C-D) Escala Yertical $1: 1.800$
Escala Harizontal $1: 3.500$

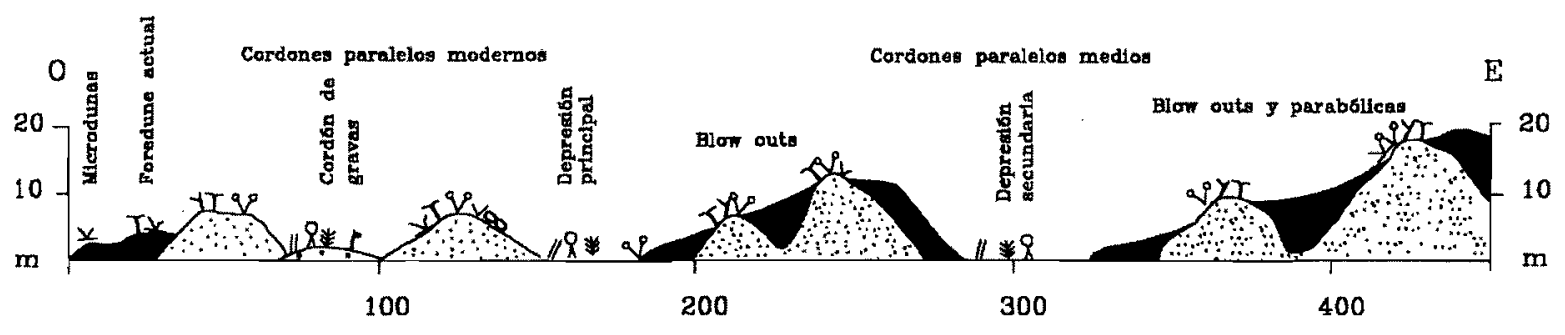

Fig. 2. Perfiles fitogeográficos del área de èstudio. Fig. 2. Phytogeographycal profiles of the study area.

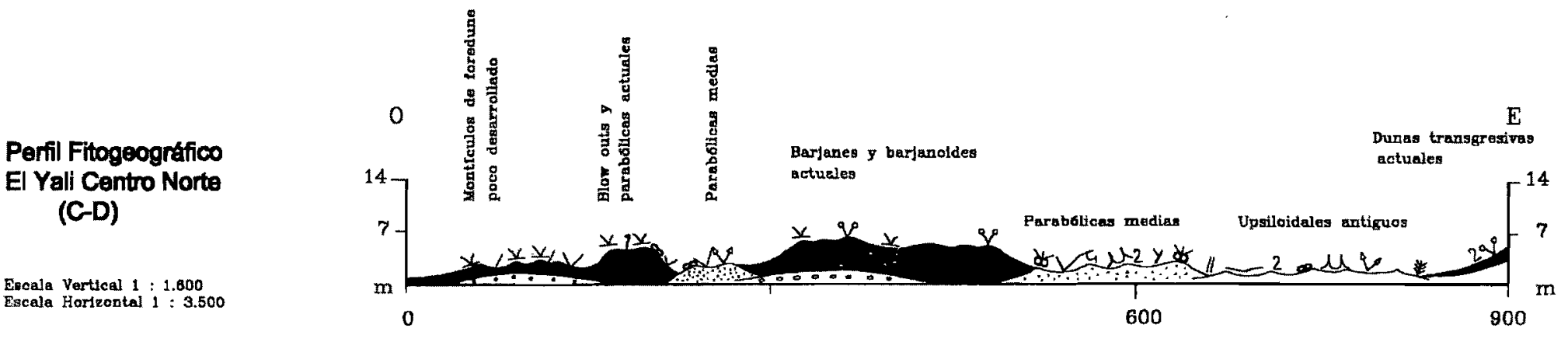



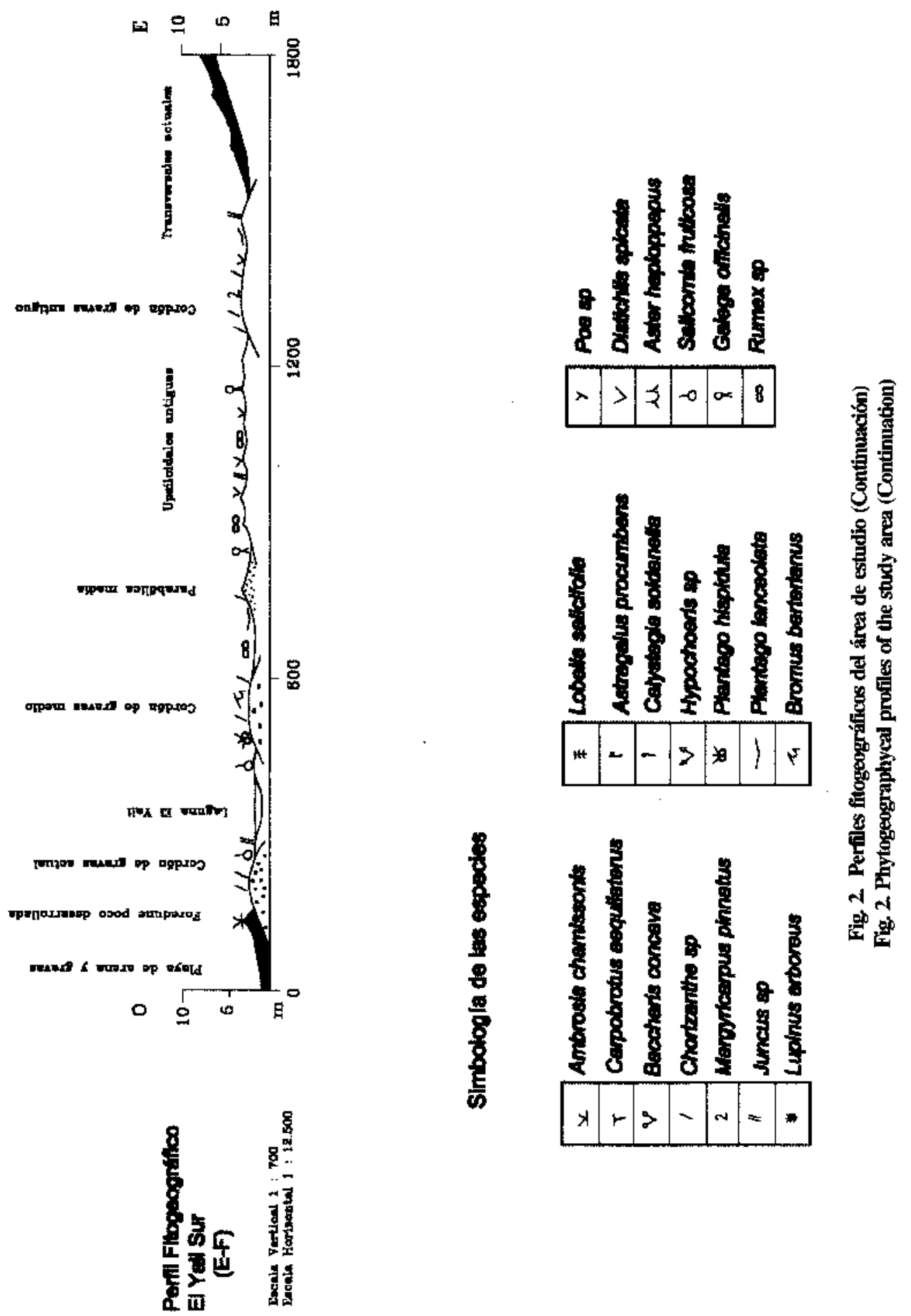

8
8
8
8
8
$\frac{8}{8}$
$\frac{5}{8}$
$\frac{8}{8}$
$\frac{1}{6}$ 
definida con el nombre de UPGMA (Unweighted Pair Group Method Using Arithmetic Averages, SNEATH \& SOKAL 1973).

\section{Concepto de Trasmudación}

Este concepto desarrollado por ARAYAVERGARA (1986), indica que los cambios de forma de la duna son generados por transportes de masa. La evolución de las formas, por lo tanto, se presenta como una sucesión de estadios dunares en la forma de un continuum de cambios morfológicos. Este continuum esta constituido por las siguientes fases: duna anterior, blow-out, duna paralela, duna upsiloidal, duna longitudinal (o duna transgresiva).

\section{RESULTADOS}

\section{Riqueza y cobertura de especies}

\section{Sistema Santo Domingo}

En el sistema Santo Domingo (Fig. 2) predomina una vegetación de tipo herbácea, salvo Baccharis concava que corresponde a la única especie leñosa que se desarrolla en estos

TABLA 1

Cobertura y Número de Especies por Categoría Dunar (\%)

(iniciales explicadas en el texto)

\begin{tabular}{|l|l|l|l|l|l|l|l|l|}
\hline \multicolumn{1}{|c|}{ Especie } & MIC & CPA & CPMO & CPME & PM & \multicolumn{1}{|c|}{ PA } & UA & CG \\
\hline Ambrossia chamissonis & $4-6$ & $2-21$ & $3-<1$ & & & & & \\
\hline Carpobrotus aequilaterus & & $35-8$ & $1-0$ & $6-6$ & & & & \\
\hline Baccharis concava & & & $25-35$ & $35-01$ & & & & \\
\hline Poa sp & & & $1-1$ & $<1-4$ & $0-5$ & & & \\
\hline Distichlis spicata & & & $1-<1$ & & & $25-25$ & & \\
\hline Calytegia soldanella & & & & & & & & \\
\hline Margyricarpus pinnatus & & & & & $3-3$ & $<1-<1$ & & \\
\hline Chorizanthe sp & & & & & & & & $25-5$ \\
\hline Bromus berterianus & & & & & $3-3$ & $<1-<1$ & & $3-<1$ \\
\hline Plantago hispidula & & & & & $5-5$ & $<1-5$ & & $5-<1$ \\
\hline Rumex sp & & & & & $5-25$ & $5-5$ & $5-5$ & \\
\hline Polygonum sanguinaria & & & & & $3-5$ & & $<1-0$ & \\
\hline Aster haplapappus & & & & & 3 & $0-5$ & & \\
\hline Plantago lanceolata & & & & & & & $25-25$ & \\
\hline Juncus sp & & & & & & $<1-5$ & $3-3$ & \\
\hline Hypochoeris radicata & & & & & & $<1-5$ & $5-5$ & $<1$ \\
\hline Oenethera stricta & & & & & & $<1-<1$ & & \\
\hline Trifolium polymorfum & & & & & & & $<1-0$ & \\
\hline Dichondra sericea & & & & & & & $<1-0$ & \\
\hline
\end{tabular}

Tabla 1. Cobertura $(\%)$ de las especies dominantes del área de estudio considerando dos muestras para cada tipo y generación de duna y de cordón muestreado. MIC: Microdunas.

CPA: Cordón Paralelo Actual. CPMO: Cordón Paralelo Moderno. CPME: Cordón

Paralelo Medio. PM: Parabólica Media. PA: Parabólica Antigua. UA: Upsiloidales Antiguas. CG: Cordón de Gravas Antiguo.

Table 1. Coverage (\%) of main species of the study area, considering two samples for each dune and ridge type and generation sampled. MIC: Microdunes. CPA: Present Foredune.

CPMO: Modern Dune Ridge. CPME: Middle Dune Ridge. PM: Medium Parabolic. PA:

Ancient Parabolic. UA: Ancient Upsiloidal. CG: Ancient Gravel Ridge. 


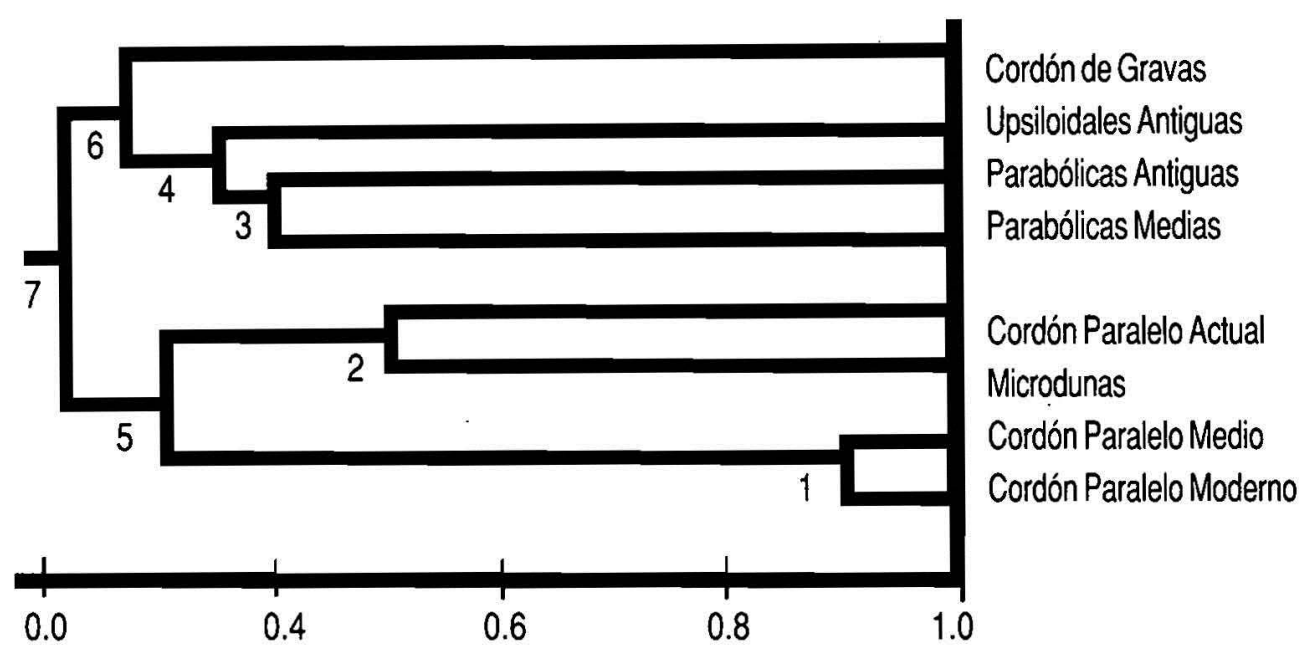

\section{Coeficiente de Similitud}

Fig. 3. Dendrograma generado a partir de análisis de agrupamientos.

Fig. 3. Cluster analysis dendrogram.

cordones. En los cordones de generación media, actualmente transgredidos por blow-outs y dunas parabólicas actuales, sobresalen montículos remanentes con vegetación.

Siguiendo un perfil transversal oeste-este, a este subsistema (Fig. 2), existe un aumento en la riqueza de especies desde los cordones más exteriores (hacia la playa) hasta los más interiores (Tabla 1). Este aumento de riqueza va desde dunas casi mono específicas como es el caso de microdunas, colonizadas por Ambrosia chamissonis (coberturas $<60 \%$ ), duna anterior actual colonizado por $A$. chamissonis y Carpobrotus aequilaterus (coberturas $<21 \%$ $\mathrm{y}<35 \%$ respectivamente) hasta los cordones de generación moderna y media (cordón paralelo moderno y cordón paralelo medio) que presentan un mayor número de especies (entre 3 y 5 especies) dentro de las que destacan $B$. coñcava, $C$. aequilaterus y Poa sp (coberturas de $<35 \%$, $<6 \%$ y $<4 \%$, respectivamente).

Las depresiones interdunares corresponden a áreas de concentración de humedad por lo que constituyen ambientes más húmedos que los cordones paralelos, lo que posibilita el desarrollo de especies de tendencia más higrófita como son Lupinus arboreous, Galega officinalis y Juncus sp. presenta la fisonomía de una pradera de hierbas y arbustos bajos, en general $<30 \mathrm{~cm}$, destacando los géneros Rumex, Distichlis, Plantago y Bromus entre las hierbas y los géneros Baccharis y Margyricarpus entre los arbustos.

En los límites orientales, tanto de este sistema como en el de Santo Domingo, en la zona de contacto con los bordes de la terraza alta, aparecen géneros con un carácter más continental como, por ejemplo, Berberis, Schinus y Maytenus.

Los resultados obtenidos permiten establecer que las dunas muestreadas en este sistema tienen mayor riqueza de especies que en el caso de Santo Domingo (Tabla 1). Dentro de las dunas y cordones de rodados muestreados, las dunas parabólicas medias son las que presentan mayor riqueza, con 9 especies. El resto presenta un rango variable entre tres y siete especies. Dominan las especies Rumex sp y Plantago hispidula en parabólicas medias (coberturas de $<25 \%$ y $5 \%$ respectivamente), Rumex $s p$ y Distichlis spicata en parabólicas antiguas y Rumex sp, Plantago lanceolata e Hypochoeris radicata en upsiloidales antiguas.

En cuanto al cordón de gravas, la especie Chorizanthe $s p$ constituye la especie dominante, con coberturas entre 25 y $50 \%$. Esta especie no fue encontrada en otros tipos de dunas. 


\section{Análisis de similitud y agrupamientos}

El análisis de agrupamientos realizado a partir del coeficiente de similitud entre las comunidades de los distintos tipos y generaciones de dunas muestreadas, permite reconocer dos grandes agrupamientos (Fig. 3), uno formado por las comunidades de los cordones paralelos de Santo Domingo y otro formado por las dunas del sistema El Yali, situación que puede indicar la existencia de distintos ensambles o comunidades de plantas para cada uno de estos sistemas dunares.

En el caso del sistema de Santo Domingo se reconocen dos subgrupos. El primero de estos, identificado con 1, está formado por el cordón paralelo moderno y medio (Fig. 3) con un coeficiente de similitud del $90 \%$. El otro, indicado con 2, está formado por las microdunas y cordón paralelo actual, con un coeficiente de similitud del $50 \%$.

Para el sistema El Yali, el análisis de agrupamientos realizado, indica 3 subgrupos, todos con coeficientes de similitud $<40 \%$. Dentro de estos, la estructura comunitaria del cordón de gravas, presenta un muy bajo valor de similitud con el resto, $>20 \%$. El resto de los subgrupos corresponden al agrupamiento 3 que reúne a las dunas parabólicas medias y parabólicas antiguas, y 4 que corresponde a las dunas upsiloidales antiguas el que se agrupa con el subgrupo anterior en valores de similitud cercanos al $30 \%$.

\section{DISCUSION}

\section{Similitud comunitaria y cronosecuencias}

Este estudio ha demostrado que las comunidades de plantas del sistema Santo Domingo y El Yali no presentan similitud en su estructura y composición. El análisis de agrupamientos realizado separa dos grandes agrupamientos, uno para comunidades en dunas del tipo primarias en el sistema Santo Domingo (microdunas y cordones paralelos) y otro para dunas del tipo secundarias (parabólicas y upsiloidales de generación media y antigua) y cordón de gravas, en el sistema El Yali. Lo anterior es una primera evidencia de la posible influencia de la morfogénesis dunar y las comunidades de plantas presentes en determinado estadio de evolución de la duna.
El aumento en la complejidad de las comunidades, a través del incremento en la riqueza de especies y coberturas conforme se avanza en la cronosecuencia, se debe posiblemente a la mayor estabilidad de las arenas y a los incrementos en los contenidos de materia orgánica, humedad y nutrientes de los arenosoles, condición que concuerda con los resultados encontrados por SEREY et al. (1976), en las dunas de Concón (Chile central).

\section{Sistema Santo Domingo}

En Santo Domingo los dos sub grupos obtenidos a partir del análisis de agrupamientos, microdunas/cordón paralelo actual y cordón paralelo moderno/cordón paralelo medio, separan claramente dos ambientes dunares distintos: a) los ambientes post-playa y duna anterior y b) los ambientes de cordones interiores. Ambos tipos de ambientes están representados por comunidades particulares definiendo una primera secuencia sucesional: un estadio temprano correspondiente con estratos herbáceos claramente de tendencia psamófila, responsables de la acreción de arena y generación de dunas anteriores, y un estadio posterior representado por estratos arbustivos $y$ pastos, en cordones interiores. Lo anterior concuerda con lo presentado en la literatura como sucesiones primarias (OLSON 1958; LICHTER 1998).

El primer subgrupo, contiene comunidades con similitudes de un $50 \%$, representado por $A$. chamisonis y C. aequilaterus, que corresponden a especies formadoras de dunas anteriores o foredunes.

Ambas especies presentan una serie de adaptaciones fisiológicas, que les permiten habitar en dunas activas; por ejemplo, rizomas verticales, raíces adventicias y tolerancia al agua con contenidos importantes de sal (KOHLER y WEISER 1966; DISRAELI 1984; MAUN 1996). Desde el punto de vista fisiológico, en este tipo de condiciones ambientales, la forma de distribuir o asignar la energía por parte de la planta, podría estar altamente influida por el ingreso de arena, razón por la cual la energía asignada a crecimiento debiera constituir el compromiso más importante de la planta.

El segundo sub grupo, correspondiente a cordón 
paralelo moderno/cordón paralelo medio, cierra la secuencia y agrupa comunidades con un $90 \%$ de similitud, lo que indica que no se han producido diferenciaciones importantes en su estructura, ya sea a través de un cambio en la composición de especies o mediante un cambio significativo de sus abundancias.

El paso del primer al segundo estadio se produce debido a la facilitación (CONNELL y SLATYER 1977) que las especies tempranas, A. chamissonis y C. aequilaterus, permiten para el establecimiento de especies más tardías como B. concava. La facilitación como mecanísmo para explicar el cambio de la vegetación en esta cronosecuencia, puede darse una vez que la duna comienza a crecer y adquiere mayor estabilidad por el efecto de estas plantas pioneras.

Los resultados obtenidos para el sistema Santo Domingo, permiten indicar que el recambio de especies como condición para el cambio sucesional es más notorio en los primeros estadios de la cronosecuencia (microdunas/ cordón paralelo actual). La alta similitud en la estructura comunitaria del cordón paralelo moderno y el cordón paralelo medio es un indicativo de que se trata de una misma comunidad.

\section{Sistema El Yali}

Como ya se indicó, las dunas muestreadas en este sistema, corresponden a fases de deflación, por lo que, comparativamente, tienen baja macicez. Este aspecto general, conjuntamente con la mayor evolución de las arenas (que está en relación directa con la presencia de vegetación) constituyen los factores que posibilitan la colonización de especies de carácter más higrófilo, por ejemplo, de los géneros Hypochoeris, Juncus, Trifolium y Polygonum, todas ellas con coberturas $<5 \%$.

El bajo valor de similitud obtenido, permite indicar diferencias en la composición y abundancia de las especies constituyentes para cada uno de estos tipos y generaciones de dunas y cordón de gravas. Este resultado indica estructuras comunitarias particulares a cada estadio. Es decir, el cambio morfológico y la diferenciación cronológica de la duna tiene su expresión en comunidades o ensambles particulares a cada uno de ellos.
El bajo porcentaje de similitud $(<10 \%)$ del cordón de gravas, indica que se trata de una comunidad particular al resto de las comunidades de dunas analizadas.

De acuerdo con lo anterior, la cronosecuencia analizada para dunas del tipo secundarias (dunas paralelas medias, dunas paralelas antiguas y dunas upsiloidales antiguas), no sólo opera en el sentido de diferenciación morfológica o de composición de las arenas (por ejemplo, en los contenidos crecientes de la fracción limo- arcilla con la edad de la duna), sino que existen estructuras comunitarias particulares a cada estadio.

Los procesos geomorfológicos son los responsables del cambio desde sucesiones primarias a sucesiones secundarias. Esto, debido a su contribución al cambio morfológico y cronológico de la duna, al pasar desde fases de acreción de arena con balances de masa positivos (construcción de cordones paralelos) a fases de deflación y balances de masa negativos. En esta situación, la baja macicez de estas dunas es una condición que propicia el paulatino establecimiento de especies invasoras de tipo herbáceo, de amplia distribución, que se localizan mayoritariamente sobre la terraza baja, como $P$. lanceolata $o$ Rumex sp. De acuerdo con esto, las causas que contribuyen al cambio sucesional en estos estadios de la cronosecuencia, estarían referidos principalmente con la disponibilidad de especies y sus características.

\section{CONCLUSIONES}

Los resultados conseguidos en este trabajo, permiten concluir lo siguiente:

- Existe una clara correspondencia entre tipos y generaciones de dunas y las comunidades de plantas que en ellos se observan, incluyendo al cordón de gravas. El patrón general es de similitudes decrecientes conforme se avanza en la cronosecuencia. La morfogénesis dunar como mecanismo que contribuye al cambio sucesional, es un factor importante en la determinación de la vía o curso de la sucesión.

- Los estadios tempranos en sucesiones primarias como los observados en microdunas, se caracterizan por la presencia de especies con 
adaptaciones físiológicas particulares, en las que la asignación de energía a crecimiento, reproducción y mantención, puede estar gobernada en forma importante por variaciones en los aportes de arena.

- La cronosecuencia dunar representa un complejo gradiente ambiental, en el cual las especies se encuentran sometidas a características ambientales particulares. Las fases tempranas están caracterizadas por restricciones ambientales, mientras que en las fases posteriores las interacciones biológicas podrían ser más importantes para explicar la estructura de las comunidades.

- La presencia de especies pioneras en los estadios tempranos de la cronosecuencia (microduna y duna anterior), facilitan, a través de la construcción de la duna y estabilización de las arenas, el establecimiento de especies más tardías. En la fase de dunas parabólicas y upsiloidales dentro de la cronosecuencia, la dominancia de las especies está relacionada, fundamentalmente, con habilidades competitivas propias de algunass especies presentes. Este hecho, dificulta caracterizar para estos estadios dunares algún patrón de reemplazo de especies.

\section{AGRADECIMIENTOS}

Mis agradecimientos por su contribución al desarrollo y finalización de este trabajo, a los profesores José Araya del Departamento de Geografía y Luis Faúndez del Departamento de Fitotecnia, ambos de la Universidad de Chile. De igual manera mis agradecimientos por las revisiones y comentarios, al doctor Luis Contreras y al profesor Yerko Vilina de la Universidad Santo Tomás, quién además, ha contribuido a divulgar la importancia ecológica de la Reserva El Yali.

\section{REFERENCIAS}

ARAYA-VERGARA, J.F., 1986. The evolution of modern coastal dune systems in central Chile. In: Gardiner, V. (Ed.), International Geomorphology, Part II, J. Wiley \& Sons: 12311242.
ARAYA-VERGARA, J.F., 1989. Remnant Coastal Dunes and Their Significance in Chilean Ergs. In: Seuffert (Ed.), Second International Conference on Geomorphology. Geoöko Plus, Vol. 1, Darmstadt: 15.

ARMESTO, J. y S.T. PICKETT, 1986. Removal experiments to test mechanisms of plant succession in oldfields. Vegetatio, 66: 85-93.

CALDICHOURY, R., 1990. La Fitogeografía del Erg Santo Domingo-El Convento. Tesis para optar al título de Geógrafo. Universidad de Chile. Inédito.

CONNELL, J.H. y R.O. SLATYER, 1977. Mechanimims of sucession in natural communities and their role in community satability and organization. American Naturalist, 111: 119-144.

DISRAELI, D. J., 1984. The effect of sand deposits on the growth and morphology of Ammophila breviligulata. Journal of Ecology, 72: 145-154.

HORN, H.S., 1966. Measurement of "overlap" in comparative ecological studies. American Natururalist, 100: 419-424.

KOHLER, A. y P. WEISSER, 1966. Contribución al problema de los neófitos: Ambrosia chamissonis (Less.) Greene en Chile. Bol. Univ. de Chile, 69 - 70:62 - 68.

LICHTER, J., 1998. Primary Succession and Forest Development on Coastal lake Michigan Sand Dunes. Ecological Monographs, 68 (4): 487-509.

MAUN, M.A., 1996. The effects of burial by sand on survival and growth of Callamovilfa longifolia. Ecoscience, 3: 93-100.

OLSON, J.S., 1958. Rates of sucession and soil cahnges on southern Lake Michigan sand dunes. Bot. Gazette, 119: 125-70.

PICKETT, S.T., S.L. COLLINS y J.J. ARMESTO, 1987. A hierarchical consideration of cauces and mechanism of succession. Vegetation, 69: 109-114. 
PICKETT, S.T., 1989. Space for time substitution as an alternative to long-term studies. In: Likens G.E. (Ed.), Long-term studies in Ecology. Springer -Verlag, New York: 110135.

SEREY, I., C. SILLARD, N. PIZARRO y J. RODRIGUEZ, 1976. Diversidad de la vegetación en las dunas de Concón. An. Mus. Hist. Nat. 9: 23-27.

SNEATH, P.H.A., y R.R. SOKAL, 1973. Numerical taxonomy: the principles and practice of numerical classification. W.H. Freeman, San Francisco.
VAN DER MAAREL, E., BOOT, R.,VAN DORP, D. y RIJNTJES, J., 1985. Vegetation sucession on the dunes near Oostvoorne, The Netherlands: A comparison of the vegetation in 1959 and 1980. Vegetatio, 58: 137-87.

WALKER, L. R. y CHAPIN, F.S., 1987. Interactions among processes controling successional cahnge. Oikos, 50: 131-135.

\section{Imagen de Investigaciones GEOGRíflens}

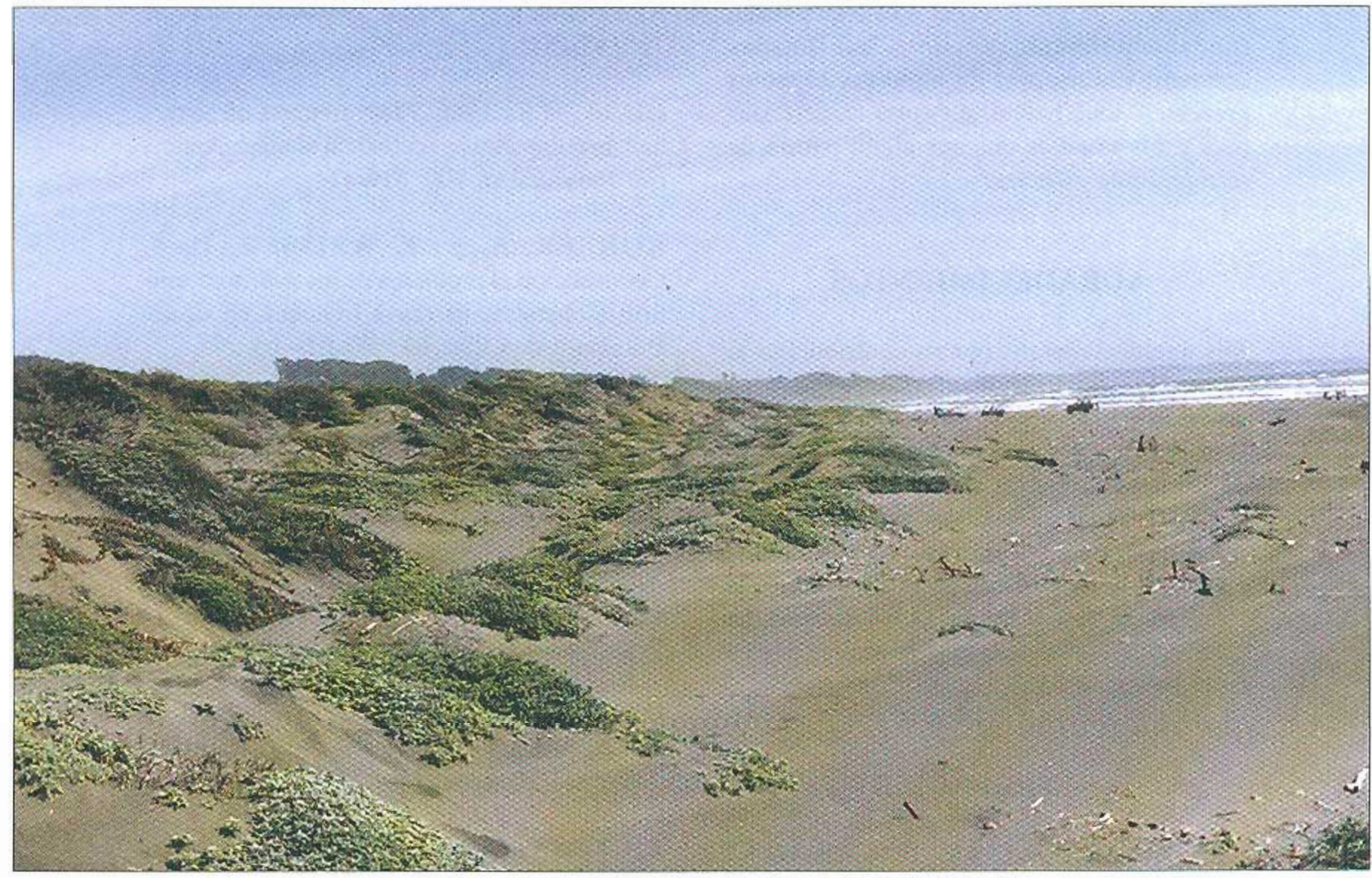

Foto 2: Comunidades vegetales asociadas a cordones de duna anterior en Santo Domingo (Chile Central - Costa de Santiago): cordón externo con Ambrosia chamissonis; cordón interno con Ambrosia chamissonis y Carpobrotus aequilaterus (Foto J.F. Araya Vergara). 